\title{
Coefficients Bounds for a General Subclasses of m-Fold Symmetric Bi-Univalent Functions
}

\author{
Waggas Galib Atshan \\ Department of Mathematics \\ College of Computer Science \\ and Information Technology
}

University of Al-Qadisiyah, Diwaniya-Iraq

E-mail: waggas.galib@qu.edu.iq

\author{
Najah Ali Jiben Al-Ziadi \\ Department of Mathematics \\ College of Science \\ University of Baghdad, Baghdad -Iraq \\ E-mail: najah.ali@qu.edu.iq
}

Recived : 7\5\2017 Revised : 19\6\2017

Accepted : 9\7\2017

\begin{abstract}
In this paper, we introduce and investigate a new general subclasses $\mathcal{R}_{\Sigma_{m}}(\tau, \lambda, \eta ; \alpha)$ and $\mathcal{R}_{\Sigma_{m}}(\tau, \lambda, \eta ; \beta)$ of $\Sigma_{m}$ consisting of analytic and $\mathrm{m}$-fold symmetric bi-univalent functions in the open unit disk $U$. We obtain estimates on the Taylor-Maclaurin coefficients $\left|a_{m+1}\right|$ and $\left|a_{2 m+1}\right|$. Also, we obtain new special cases for our results.
\end{abstract}

Keywords: Analytic function, Univalent function, Bi-Univalent function, m-Fold symmetric function, m-Fold symmetric bi-univalent function.

Mathematics subject classification : 30C45. 30C50. $30 \mathrm{C} 80$.

\section{Introduction}

Symbolized by $\mathcal{A}$ the class of functions of the form:

$f(z)=z+\sum_{n=2}^{\infty} a_{n} z^{n}$

which are analytic in the open unit disk $U=$ $\{z: z \in \mathbb{C}$ and $|z|<1\}$. Also, symbolized by $\mathcal{S}$ the class of all functions in $\mathcal{A}$ which are univalent and normalized by $f(0)=0=f^{\prime}(0)-1$ in $U$. The wellinvestigated subclasses of the univalent function class $\mathcal{S}$ are the class of starlike functions of order $\alpha(0 \leq \alpha<$ 1 ), symbolized by $\mathcal{S}^{*}(\alpha)$ and the class of convex functions of order $\alpha$ symbolized by $\mathcal{K}(\alpha)$ in $U$.

The Koebe One-Quarter Theorem [1] shows that the image of $U$ under every function $f$ from $\mathcal{S}$ contains a disk of radius 1/4. Thereby every such univalent function has an inverse $f^{-1}$ which satisfies

$f^{-1}(f(z))=z \quad(z \in U)$

and

$$
f\left(f^{-1}(w)\right)=w \quad\left(|w|<r_{0}(f) ; r_{0}(f) \geq \frac{1}{4}\right)
$$

where

$f^{-1}(w)=g(w)=w-a_{2} w^{2}+\left(2 a_{2}^{2}-a_{3}\right) w^{3}-\left(5 a_{2}^{3}\right.$

$\left.-5 a_{2} a_{3}+a_{4}\right) w^{4}+\cdots$.

The function $f \in \mathcal{A}$ is considered bi-univalent in $U$ if both $f$ and $f^{-1}$ are univalent in $U$. Given by the TaylorMaclaurin series expansion (1.1), the class of all biunivalent functions in $U$ can be symbolized by $\Sigma$.

For each function $f \in \mathcal{S}$, the function

$h(z)=\sqrt[m]{f\left(z^{m}\right)}(z \in U, m \in \mathbb{N})$

is univalent and the unit disk $U$ can be mapped into a region with $\mathrm{m}$-fold symmetry. It is considered $\mathrm{m}$-fold symmetric (see $[2,3]$ ) if the function has the following normalized form:

$f(z)=z+\sum_{k=1}^{\infty} a_{m k+1} z^{m k+1} \quad(z \in U, m \in \mathbb{N})$. 
The class of $\mathrm{m}$-fold symmetric univalent functions in $U$, which are normalized by the series expansion (1.4) can be symbolized by $\mathcal{S}_{m}$ and the functions in the class $\mathcal{S}$ are one-fold symmetric (that is, $\mathrm{m}=1$ ).

In [4] Srivastava et al. specified that $\mathrm{m}$-fold symmetric bi-univalent function analogues to the concept of $\mathrm{m}$-fold symmetric univalent functions and these gave some important results, such as each function $f \in \Sigma$ generates an m-fold symmetric bi-univalent function for each $m \in \mathbb{N}$, in their study. As for as the normalized form of $f$ given by (1.4) is concerned, they obtained the series expansion for $f^{-1}$ as follows:

$f^{-1}(w)=g(w)=w-a_{m+1} w^{m+1}$

$+\left[(m+1) a_{m+1}^{2}-a_{2 m+1}\right] w^{2 m+1}-$

$\left[\frac{1}{2}(m+1)(3 m+2) a_{m+1}^{3}-(3 m+2) a_{m+1} a_{2 m+1}+a_{3 m+1}\right]$ $w^{3 m+1}+\cdots$,

where $f^{-1}=g$. The class of $\mathrm{m}$-fold symmetric biunivalent functions in $U$ can be symbolized by $\Sigma_{m}$. For $m=1$, formula (1.5) coincides with formula (1.2) of the class $\Sigma$.

Mathematicians such as Lewin [5], Brannan and Clunie [6] as well as Netanyahu [7] studied the functions in $\Sigma$ and proved the following, the first investigated the bi-univalent function class $\Sigma$ and showed that $\left|a_{2}\right|<1.51$, the second showed that $\left|a_{2}\right|<\sqrt{2}$, whereas the third showed that $\max \left|a_{2}\right|=$ $4 / 3$ if $f(z) \in \Sigma$, but the best known estimate for functions in $\Sigma$ were obtained by Tan [8] in 1984, that is, $\left|a_{2}\right| \leq 1.485$. The coefficient estimate problem involving the bound of $\left|a_{2}\right|(n \in \mathbb{N} \backslash\{1,2\})$ for each $f \in \Sigma$ given by (1.4) is still an open problem. In fact, the aforecited work of Srivastava et al. [9] essentially revived the investigation of various subclasses of the biunivalent function class $\Sigma$ in recent years. Recently, many authors investigated bounds for various subclasses of bi-univalent functions (see[10, 11, 12, 13, 14, 9, $15])$.
The object of the present paper is to obtain estimates on the Taylor-Maclaurin coefficients $\left|a_{m+1}\right|$ and $\left|a_{2 m+1}\right|$ for functions belonging to the new general subclasses $\mathcal{R}_{\Sigma_{m}}(\tau, \lambda, \eta ; \alpha)$ and $\mathcal{R}_{\Sigma_{m}}(\tau, \lambda, \eta ; \beta)$ of $\Sigma_{m}$. Also, some interesting applications of the results presented here are also discussed.

In order to derive our main result, we have to recall here the following lemma [1].

Lemma 1.1. If $h \in \mathcal{P}$, then $\left|c_{k}\right| \leq 2$ for each $k \in \mathbb{N}$, where $\mathcal{P}$ is the family of all functions $h$, analytic in $U$, for which

$R(h(z))>0 \quad(z \in U)$,

where

$h(z)=1+c_{1} z+c_{2} z^{2}+\cdots \quad(z \in U)$.

\section{Coefficients bounds for the function class}

$\mathcal{R}_{\Sigma_{m}}(\tau, \lambda, \eta ; \alpha)$

Definition 2.1. A function $f \in \Sigma_{m}$ given by (1.4) is said to be in the class $\mathcal{R}_{\Sigma_{m}}(\tau, \lambda, \eta ; \alpha)$ if the following conditions are satisfied:

$\left|\arg \left(1+\frac{1}{\tau}\left[(1-\lambda) \frac{f(z)}{z}+\lambda f^{\prime}(z)+\eta z f^{\prime \prime}(z)-1\right]\right)\right|$

$<\frac{\alpha \pi}{2} \quad(z \in U)$

and

$\left|\arg \left(1+\frac{1}{\tau}\left[(1-\lambda) \frac{g(w)}{w}+\lambda g^{\prime}(w)+\eta w g^{\prime \prime}(w)-1\right]\right)\right|$

$<\frac{\alpha \pi}{2}(w \in U)$,

where $(\tau \in \mathbb{C} \backslash\{0\} ; \lambda \geq 1 ; 0 \leq \eta \leq 1 ; 0<\alpha \leq 1)$ and the function $g$ is given by (1.5).

Theorem 2.1. Let the function $f(z)$, given by (1.4), be in the class $\mathcal{R}_{\Sigma_{m}}(\tau, \lambda, \eta ; \alpha)$. Then

$\left|a_{m+1}\right| \leq$

$\frac{2 \alpha|\tau|}{\sqrt{\left|\alpha \tau(m+1)(1+2 \lambda m+2 \eta m(2 m+1))+(1-\alpha)(1+\lambda m+\eta m(m+1))^{2}\right|}}$ 
and

$$
\begin{aligned}
& \left|a_{2 m+1}\right| \leq \frac{2 \alpha^{2}|\tau|^{2}(m+1)}{(1+\lambda m+\eta m(m+1))^{2}} \\
& +\frac{2 \alpha|\tau|}{(1+2 \lambda m+2 \eta m(2 m+1))}
\end{aligned}
$$

Proof. Let $f \in \mathcal{R}_{\Sigma_{m}}(\tau, \lambda, \eta ; \alpha)$. Then

$1+\frac{1}{\tau}\left[(1-\lambda) \frac{f(z)}{z}+\lambda f^{\prime}(z)+\eta z f^{\prime \prime}(z)-1\right]$

$=[p(z)]^{\alpha}$

and

$1+\frac{1}{\tau}\left[(1-\lambda) \frac{g(w)}{w}+\lambda g^{\prime}(w)+\eta \mathrm{w} g^{\prime \prime}(w)-1\right]$

$$
=[q(w)]^{\alpha} \text {, }
$$

where $g=f^{-1}, p(z), q(z)$ in $\mathcal{P}$ and have the forms

$p(z)=1+p_{m} z^{m}+p_{2 m} z^{2 m}+p_{3 m} z^{3 m}+\cdots$

and

$q(w)=1+q_{m} w^{m}+q_{2 m} w^{2 m}+q_{3 m} w^{3 m}+\cdots$.

Now, equating the coefficients in (2.5) and (2.6), we get

$$
\begin{aligned}
& \left(\frac{1+\lambda m+\eta m(m+1)}{\tau}\right) a_{m+1}=\alpha p_{m} \\
& \left(\frac{1+2 \lambda m+2 \eta m(2 m+1)}{\tau}\right) a_{2 m+1} \\
& =\alpha p_{2 m}+\frac{1}{2} \alpha(\alpha-1) p_{m}^{2} \\
& -\left(\frac{1+\lambda m+\eta m(m+1)}{\tau}\right) a_{m+1}=\alpha q_{m}
\end{aligned}
$$

and

$\left(\frac{1+2 \lambda m+2 \eta m(2 m+1)}{\tau}\right)\left[(m+1) a_{m+1}^{2}-a_{2 m+1}\right]$

$=\alpha q_{2 m}+\frac{1}{2} \alpha(\alpha-1) q_{m}^{2}$.

From (2.9) and (2.11), we find

$$
p_{m}=-q_{m}
$$

and

$$
\begin{aligned}
& 2\left(\frac{1+\lambda m+\eta m(m+1)}{\tau}\right)^{2} a_{m+1}^{2} \\
& =\alpha^{2}\left(p_{m}^{2}+q_{m}^{2}\right) .
\end{aligned}
$$

From (2.10), (2.12) and (2.14), we get $\left(\frac{1+2 \lambda m+2 \eta m(2 m+1)}{\tau}\right)(m+1) a_{m+1}^{2}$

$$
\begin{aligned}
& =\alpha\left(p_{2 m}+q_{2 m}\right)+\frac{\alpha(\alpha-1)}{2}\left(p_{m}^{2}+q_{m}^{2}\right) \\
& =\alpha\left(p_{2 m}+q_{2 m}\right) \\
& +\frac{(\alpha-1)}{\alpha}\left(\frac{1+\lambda m+\eta m(m+1)}{\tau}\right)^{2} a_{m+1}^{2} .
\end{aligned}
$$

Therefore, we have

$a_{m+1}^{2}=$

$\frac{\alpha^{2} \tau^{2}\left(p_{2 m}+q_{2 m}\right)}{\alpha \tau(m+1)(1+2 \lambda m+2 \eta m(2 m+1))+(1-\alpha)\left((1+\lambda m+\eta m(m+1))^{2}\right.}$.

Applying Lemma (1.1) for the coefficients $p_{2 m}$ and $q_{2 m}$, we immediately have

$\left|a_{m+1}\right| \leq$

$\frac{2 \alpha|\tau|}{\sqrt{\left|\alpha \tau(m+1)(1+2 \lambda m+2 \eta m(2 m+1))+(1-\alpha)(1+\lambda m+\eta m(m+1))^{2}\right|}}$

The last inequality gives the desired estimate on $\left|a_{m+1}\right|$ given in (2.3).

Next, in order to find the bound on $\left|a_{2 m+1}\right|$, by subtracting (2.12) from (2.10), we obtain

$$
\begin{aligned}
& 2\left(\frac{1+2 \lambda m+2 \eta m(2 m+1)}{\tau}\right) a_{2 m+1} \\
& -\left(\frac{1+2 \lambda m+2 \eta m(2 m+1)}{\tau}\right)(m+1) a_{m+1}^{2} \\
& =\alpha\left(p_{2 m}-q_{2 m}\right)+\frac{\alpha(\alpha-1)}{2}\left(p_{m}^{2}-q_{m}^{2}\right) .
\end{aligned}
$$

It follows from (2.13), (2.14) and (2.18) that

$$
\begin{aligned}
& a_{2 m+1}=\frac{\alpha^{2} \tau^{2}(m+1)\left(p_{m}^{2}+q_{m}^{2}\right)}{4(1+\lambda m+\eta m(m+1))^{2}} \\
& +\frac{\alpha \tau\left(p_{2 m}-q_{2 m}\right)}{2(1+2 \lambda m+2 \eta m(2 m+1))} .
\end{aligned}
$$

Applying Lemma (1.1) once again for the coefficients $p_{m}, p_{2 m}, q_{m}$ and $q_{2 m}$, we readily get

$$
\begin{aligned}
& \left|a_{2 m+1}\right| \leq \frac{2 \alpha^{2}|\tau|^{2}(m+1)}{(1+\lambda m+\eta m(m+1))^{2}} \\
& +\frac{2 \alpha|\tau|}{(1+2 \lambda m+2 \eta m(2 m+1))}
\end{aligned}
$$

This completes the proof of Theorem (2.1). 


\section{Coefficients bounds for the function class}

$\mathcal{R}_{\Sigma_{m}}(\tau, \lambda, \eta ; \boldsymbol{\beta})$

Definition 3.1. A function $f \in \Sigma_{m}$ given by (1.4) is said to be in the class $\mathcal{R}_{\Sigma_{m}}(\tau, \lambda, \eta ; \beta)$ if the following conditions are satisfied:

$\operatorname{Re}\left(1+\frac{1}{\tau}\left[(1-\lambda) \frac{f(z)}{z}+\lambda f^{\prime}(z)+\eta \mathrm{z} f^{\prime \prime}(z)-1\right]\right)$

$>\beta \quad(z \in U)$

and

$\operatorname{Re}\left(1+\frac{1}{\tau}\left[(1-\lambda) \frac{g(w)}{w}+\lambda g^{\prime}(w)+\eta \mathrm{wg}^{\prime \prime}(w)-1\right]\right)$

$>\beta \quad(w \in U)$,

where $(\tau \in \mathbb{C} \backslash\{0\} ; \lambda \geq 1 ; 0 \leq \eta \leq 1 ; 0 \leq \beta<1)$ and the function $g$ is given by (1.5).

Theorem 3.1. Let the function $f(z)$, given by (1.4), be in the class $\mathcal{R}_{\Sigma_{m}}(\tau, \lambda, \eta ; \beta)$. Then

$\left|a_{m+1}\right| \leq \sqrt{\frac{4|\tau|(1-\beta)}{(m+1)(1+2 \lambda m+2 \eta m(2 m+1))}}$

and

$\left|a_{2 m+1}\right| \leq \frac{2|\tau|^{2}(1-\beta)^{2}(m+1)}{(1+\lambda m+\eta m(m+1))^{2}}$

$+\frac{2|\tau|(1-\beta)}{(1+2 \lambda m+2 \eta m(2 m+1))}$.

Proof. It follows from (3.1) and (3.2) that there exist $p, q \in \mathcal{P}$ such that

$1+\frac{1}{\tau}\left[(1-\lambda) \frac{f(z)}{z}+\lambda f^{\prime}(z)+\eta z f^{\prime \prime}(z)-1\right]$

$=\beta+(1-B) p(z)$

and

$1+\frac{1}{\tau}\left[(1-\lambda) \frac{g(w)}{w}+\lambda g^{\prime}(w)+\eta \mathrm{wg}^{\prime \prime}(w)-1\right]$

$=\beta+(1-B) q(w)$,

where $p(z)$ and $q(w)$ have the forms (2.7) and (2.8), respectively. Equating coefficients in (3.5) and (3.6), we get

$\left(\frac{1+\lambda m+\eta m(m+1)}{\tau}\right) a_{m+1}$

$=(1-\beta) p_{m}$,

$$
\begin{aligned}
& \left(\frac{1+2 \lambda m+2 \eta m(2 m+1)}{\tau}\right) a_{2 m+1} \\
& =(1-\beta) p_{2 m} \\
& -\left(\frac{1+\lambda m+\eta m(m+1)}{\tau}\right) a_{m+1}=(1-\beta) q_{m}
\end{aligned}
$$

and

$\left(\frac{1+2 \lambda m+2 \eta m(2 m+1)}{\tau}\right)\left[(m+1) a_{m+1}^{2}-a_{2 m+1}\right]$

$=(1-\beta) q_{2 m}$.

From (3.7) and (3.9), we find

$p_{m}=-q_{m}$

and

$2\left(\frac{1+\lambda m+\eta m(m+1)}{\tau}\right)^{2} a_{m+1}^{2}$

$=(1-\beta)^{2}\left(p_{m}^{2}+q_{m}^{2}\right)$.

Adding (3.8) and (3.10), we have

$\left(\frac{1+2 \lambda m+2 \eta m(2 m+1)}{\tau}\right)(m+1) a_{m+1}^{2}$

$=(1-\beta)\left(p_{2 m}+q_{2 m}\right)$.

Therefore, we obtain

$a_{m+1}^{2}=\frac{\tau(1-\beta)\left(p_{2 m}+q_{2 m}\right)}{(m+1)(1+2 \lambda m+2 \eta m(2 m+1))}$.

Applying Lemma (1.1) for coefficients $p_{2 m}$ and $q_{2 m}$, we obtain

$\left|a_{m+1}\right| \leq \sqrt{\frac{4|\tau|(1-\beta)}{(m+1)(1+2 \lambda m+2 \eta m(2 m+1))}}$.

This gives the bound on $\left|a_{m+1}\right|$ as asserted in (3.3).

In order to find the bound on $\left|a_{2 m+1}\right|$, by subtracting (3.10) from (3.8), we get

$2\left(\frac{1+2 \lambda m+2 \eta m(2 m+1)}{\tau}\right) a_{2 m+1}$

$-\left(\frac{1+2 \lambda m+2 \eta m(2 m+1)}{\tau}\right)(m+1) a_{m+1}^{2}$

$=(1-\beta)\left(p_{2 m}-q_{2 m}\right)$

or equivalently

$a_{2 m+1}=\frac{(m+1)}{2} a_{m+1}^{2}$

$+\frac{\tau(1-\beta)\left(p_{2 m}-q_{2 m}\right)}{2(1+2 \lambda m+2 \eta m(2 m+1))}$. 
Upon substituting the value of $a_{m+1}^{2}$ from (3.12), we

get

$a_{2 m+1}=\frac{\tau^{2}(1-\beta)^{2}(m+1)\left(p_{m}^{2}+q_{m}^{2}\right)}{4(1+\lambda m+\eta m(m+1))^{2}}$

$+\frac{\tau(1-\beta)\left(p_{2 m}-q_{2 m}\right)}{2(1+2 \lambda m+2 \eta m(2 m+1))}$.

Applying Lemma (1.1) once again for the coefficients

$p_{m}, p_{2 m}, q_{m}$ and $q_{2 m}$, we find

$\left|a_{2 m+1}\right| \leq \frac{2|\tau|^{2}(1-\beta)^{2}(m+1)}{(1+\lambda m+\eta m(m+1))^{2}}$

$+\frac{2|\tau|(1-\beta)}{(1+2 \lambda m+2 \eta m(2 m+1))}$.

This completes the proof of Theorem (3.1).

\section{Corollaries and Consequences}

For one-fold symmetric bi-univalent functions, the classes $\mathcal{R}_{\Sigma_{m}}(\tau, \lambda, \eta ; \alpha)$ and $\mathcal{R}_{\Sigma_{m}}(\tau, \lambda, \eta ; \beta)$ reduce to the classes $\mathcal{R}_{\Sigma}(\tau, \lambda, \eta ; \alpha)$ and $\mathcal{R}_{\Sigma}(\tau, \lambda, \eta ; \beta)$ and thus, Theorem (2.1) and Theorem (3.1) reduce to Corollary (4.1) and Corollary(4.2), respectively.

Corollary 4.1. Let $f(z)$ given by (1.1) be in the class $\mathcal{R}_{\Sigma}(\tau, \lambda, \eta ; \alpha)$. Then

$\left|a_{m+1}\right|$

$\leq \frac{2 \alpha|\tau|}{\sqrt{\left|2 \alpha \tau(1+2 \lambda+6 \eta)+(1-\alpha)(1+\lambda+2 \eta)^{2}\right|}}$

and

$\left|a_{2 m+1}\right| \leq \frac{4 \alpha^{2}|\tau|^{2}}{(1+\lambda+2 \eta)^{2}}+\frac{2 \alpha|\tau|}{(1+2 \lambda+6 \eta)}$.

Corollary 4.2. Let $f(z)$ given by (1.1) be in the class $\mathcal{R}_{\Sigma}(\tau, \lambda, \eta ; \beta)$. Then

$$
\left|a_{m+1}\right| \leq \sqrt{\frac{2|\tau|(1-\beta)}{(1+2 \lambda+6 \eta)}}
$$

and

$\left|a_{2 m+1}\right| \leq \frac{4|\tau|^{2}(1-\beta)^{2}}{(1+\lambda+2 \eta)^{2}}+\frac{2|\tau|(1-\beta)}{(1+2 \lambda+6 \eta)}$.

The classes $\mathcal{R}_{\Sigma}(\tau, \lambda, \eta ; \alpha)$ and $\mathcal{R}_{\Sigma}(\tau, \lambda, \eta ; \beta)$ are defined in the following way:

Definition 4.1. A function $f(z) \in \Sigma$ given by (1.1) is said to be in the class $\mathcal{R}_{\Sigma}(\tau, \lambda, \eta ; \alpha)$ if the following conditions are satisfied: $\left|\arg \left(1+\frac{1}{\tau}\left[(1-\lambda) \frac{f(z)}{z}+\lambda f^{\prime}(z)+\eta z f^{\prime \prime}(z)-1\right]\right)\right|$

$<\frac{\alpha \pi}{2} \quad(z \in U)$

and

$\left|\arg \left(1+\frac{1}{\tau}\left[(1-\lambda) \frac{g(w)}{w}+\lambda g^{\prime}(w)+\eta w g^{\prime \prime}(w)-1\right]\right)\right|$

$<\frac{\alpha \pi}{2} \quad(w \in U)$,

where $(\tau \in \mathbb{C} \backslash\{0\} ; \lambda \geq 1 ; 0 \leq \eta \leq 1 ; 0<\alpha \leq 1)$ and the function $g$ is given by (1.2).

Definition 4.2. A function $f(z) \in \Sigma$ given by (1.1) is said to be in the class $\mathcal{R}_{\Sigma}(\tau, \lambda, \eta ; \beta)$ if the following conditions are satisfied:

$R e\left(1+\frac{1}{\tau}\left[(1-\lambda) \frac{f(z)}{z}+\lambda f^{\prime}(z)+\eta z f^{\prime \prime}(z)-1\right]\right)$

$>\beta \quad(z \in U)$

and

$\operatorname{Re}\left(1+\frac{1}{\tau}\left[(1-\lambda) \frac{g(w)}{w}+\lambda g^{\prime}(w)+\eta w g^{\prime \prime}(w)-1\right]\right)$

$>\beta \quad(w \in U)$,

where $(\tau \in \mathbb{C} \backslash\{0\} ; \lambda \geq 1 ; 0 \leq \eta \leq 1 ; 0 \leq \beta<1)$ and the function $g$ is given by (1.2).

Remark 4.1. For m-fold symmetric bi-univalent functions, if we put $\lambda=1$ in Theorem (2.1) and Theorem (3.1), we obtain the results which were given by Srivastava et al.[16]. In addition, if we put $\eta=0$ in Theorem (2.1) and Theorem (3.1), we obtain the results which were given by Srivastave et al. [17]

Remark 4.2. For one-fold symmetric bi-univalent functions, if we put $\tau=1$ and $\lambda=1$ in Theorem (2.1) and Theorem (3.1), we obtain the results which were given by Frasin [12]. In addition, if we put $\tau=1$ and $\eta=0$ in Theorem (2.1) and Theorem (3.1), we obtain the results which were given by Frasin and Aouf [13]. 


\section{References}

[1] P. L. Duren, Univalent Functions, Grundlehren der Mathematischen Wissenschaften 259, Springer, New York, USA, (1983).

[2] W. Koepf, Coefficients of symmetric functions of bounded boundary rotations, Proc. Amer. Math. Soc., 105, 324-329, (1989).

[3] C. Pommerenke, On the coefficients of close-toconvex functions, Michigan Math. J., 9, 259-269, (1962).

[4] H. M. Srivastava, S. Sivasubramanian and R. Sivakumar, Initial coefficient bounds for a subclass of m-fold symmetric bi-univalent functions, Tbilisi Mathematical J., 7(2), 1-10, (2014).

[5] M. Lewin, On a coefficient problem for bi-univalent functions, Proc. Amer. Math. Soc., 18, 63-68, (1967), .

[6] D. Brannan and J. G. Clunie (Eds), Aspects of contemporary complex analysis, (Proceedings of the NATO advanced study institute held at the Univ. of Durham, Durham; July 1-20, 1979). New York, London: Academic Press, (1980).

[7] E. Netanyahu, The minimal distance of the image boundary from the origin and the second coefficient of a univalent function in $|z|<1$, Archive for Rotional Mechanics and Analysis, 32 (2), 100-112, (1969).

[8] D. -L. Tan, Coefficient estimates for bi-univalent functions, Chinese Ann Math Ser, A5, 559-568, (1984).
[9] H. M. Srivastava, A. K. Mishra and P. Gochhayat, Certain subclasses of analytic and bi-univalent functions, Appl. Math, Lett., 23, 1188-1192, (2010).

[10] S. Bulut, Faber polynomial coefficient estimates for a subclass of analytic bi-univalent functions, Filomat, 30 (6), 1567-1575, (2016).

[11] E. Deniz, Certain subclasses of bi-univalent functions satisfying subordinate conditions, Journal of Classical Analysis, 2(1), 49-60, (2013).

[12] B. A. Frasin, Coefficient bounds for certain classes of bi-univalent functions, Hacet. J. Math. Stat., 43(3), 383-389, (2014).

[13] B. A. Frasin and M. K. Aouf, New subclasses of bi-univalent functions, Appl. Math. Lett., 24,15691573, (2011),

[14] X. -F. Li and A. -P. Wang, Tow new subclasses of bi-univalent functions, Int. Math. Forum, 7, 1495-1504, (2012).

[15] Q.-H. Xu, Y.-C. Gui and H. M. Srivastava, Coefficient estimates for a certain subclass of analytic and bi-univalent functions, Appl. Math. Lett., 25 (6), 990-994, (2012).

[16] H. M. Srivastava, S. Gaboury and F. Ghanim, Coefficient estimates for some subclasses of $\mathrm{m}$ fold symmetric bi-univalent functions, Acta Universitatis Apulensis, 41, 153-164, (2015).

[17] H. M. Srivastava, S. Gaboury and F. Ghanim, Initial coefficient estimates for some subclasses of m-fold symmetric bi-univalent functions, Acta Mathematica Scientia, 36 B(3), 863-871, (2016). 
حدود المعاملات لاصناف جزئية عامة للاوال الثنائية التكافؤ المتناظرة المطوية من النمط

$$
\text { تجاح علي جبن الزيّة الزياديات }
$$

E-mail: najah.ali@qu.edu.iq

$$
\begin{aligned}
& \text { وقاص غالب عطشان } \\
& \text { قاسم الرياضيات } \\
& \text { كلية علوم الحاسبات وتكنولوجيا المعلومات } \\
& \text { جامعة القادسية ولكوبة }
\end{aligned}
$$

E-mail: waggas.galib@qu.edu.iq

(المستخلص : (1)

في هذا البحث، قدمنا وناقشنا اصناف جزئية عامة جديدة و المتكونة من الدو ال التحليلية الثنائية التكافؤ المتناظرة المطوية من النمط في قرص الوحدة المفتوح U . حصلنا على تخمينات حول معاملات مكلورين ـ تايلور | جديدة لنتائجنا. 